\title{
Datenschutzdialog auf dem europäischen Privacy Open Space
}

Die Erfahrungen von Entwicklern, Nutzern und Datenschutzbehörden haben gezeigt, dass die Anforderungen des Datenschutzes innerhalb aller Arten von elektronischen Diensten (e-Services) bereits im frühen Stadium berücksichtigt, umgesetzt und in Prozesse integriert werden müssen. Ein neues Projekt des ULD - das „European Privacy Open Space“, kurz „PrivacyOS“ - will dabei helfen, die Sichtweisen der verschiedenen Akteure zusammen zu bringen.

\section{Das Konzept}

Für PrivacyOS hat das ULD einen $\mathrm{Zu}$ schlag für eine Förderung im Rahmen des „ICT Policy Support Programmes“ der Europäischen Kommission bekommen. Das Projekt führt Vertreter aus den Bereichen Wirtschaft, Wissenschaft, Regierung und Gesellschaft zusammen, um die Entwicklung und die Anwendung von Datenschutzinfrastrukturen in Europa zu fördern und zu unterstützen.

Kern der Arbeit von PrivacyOS ist der Datenschutzdiskurs auf Konferenzen, die nach der sogenannten „Open Space“-Methode ausgerichtet werden: Die Teilnehmer bringen eigene Themen ein und gestalten dazu Vorträge und Diskussionen. Die Agenda eines „Open Space“ (engl. für „offener Raum“) wird erst zu Beginn der Konferenz erstellt. Jeder Teilnehmer kann ein Thema mit datenschutzrechtlichem Bezug einbringen und bekommt in Abhängigkeit des Interesses der anderen Teilnehmer einen Zeitblock („Slot“, ca. 45 Minuten) und einen Raum zugeordnet. Diese Dynamik ermöglicht es, neue und aktuelle Themen zu behandeln und $\mathrm{zu}$ diskutieren, während gleichzeitig ein Einblick in die Entwicklung in den europäischen Mitgliedsstaaten gewährt wird.

Innerhalb des Projektes PrivacyOS wird den Projektpartner und Konferenzteilnehmern die Möglichkeit geboten, sich über „Best-Practices“, datenschutzrechtliche Herausforderungen und mögliche Lösungen auszutauschen. Aus diesem Grund werden über einen Zeitraum von zwei Jahren vier Open-Space-Konferenzen parallel zu Veranstaltungen mit datenschutzrechtlicher Relevanz organisiert. Auf den PrivacyOS-Konferenzen werden eine Vielzahl von Themen wie etwa Electronic ID-Cards, eParticipation, Datenschutzsiegel oder Krypto-Mechanismen diskutiert und Anwendungsmöglichkeiten erarbeitet.

Zu den Partnern von PrivacyOS zählen u.a. Hewlett-Packard Research Labs, Bristol, ERCIM (W3C), NEC, der Dachverband Europäischer Verbraucherschutzorganisationen BEUC sowie mehrere europäische Nichtregierungsorganisationen, Universitäten und Datenschutzbeauftragte.

Ziel ist es, eine Möglichkeit zur Zusammenarbeit und zum Austausch innerhalb der Mitgliedsstaaten und den verschiedenen EU-Projekten zum Thema Datenschutz zu etablieren.

\section{PrivacyOS Konferenzen}

Die erste PrivacyOS-Konferenz wurde vom 13. bis 15. Oktober 2008 in Straßburg in den Räumen des Europäischen Parlaments zeitgleich zur 30. Internationalen Konferenz der Datenschutzbeauftragten ausgerichtet.

Die Konferenz stand unter der Schirmherrschaft Alexander Alvaro, MdEP. Schwerpunkte der ersten Konferenz lagen auf dem Bündeln von Fachkenntnis, dem Finden neuer Projekte und der Präsentation von bereits bestehenden Ergebnissen. Teilnehmer aus 11 Mitgliedsstaaten entwickelten eine Vielzahl von datenschutzrechtlichen Themen umspannende Agenda (für eine Übersicht der Präsentationen siehe www.privacyos.eu).
Die Teilnehmer diskutierten und präsentierten in 20 "Slots“ (Workshops mit einer Länge von ca. 45 Minuten) Themen wie z.B. e-Health, Standardisierung im Datenschutz, Datenschutz-Gütesiegel (EuroPriSe) und Vorratsdatenspeicherung.

Nach dem erfolgreichen Start des Projekts wurde die zweite PrivacyOS Konferenz vom 01.bis 03. April 2009 in Berlin ausgerichtet. Die Veranstaltung fand in den Räumen des MediaCenters in Berlin in Kooperation mit der Konferenz „re:publica“ (www.re-publica.de) statt und stand unter der Schirmherrschaft des Bundesbeauftragen für den Datenschutz und die Informationsfreiheit, Peter Schaar.

Über 80 Vertreter aus den Bereichen Wirtschaft, Wissenschaft, Regierung und Gesellschaft wiederum aus 11 Mitgliedsstaaten diskutierten und referierten dort. Thematisch bildete der Datenschutz in Sozialen Netzwerken einen Schwerpunkt mit Vorträgen und Diskussionen unter den Titeln. „Netiquette“ in Sozialen Netzwerken, „Human readable Privacy Policy create commons for privacy“, Schutz und Aufklärung von Minderjährigen oder „access-control“, aber auch e-Health und e-Government sowie Identity Management wurden angesprochen.

Die PrivacyOS Konferenzen adressieren IT-Rechtsexperten und IT-Techniker, Hersteller von IT-Produkten oder IT-Services sowie Datenschutzbehörden, die an einem Diskurs zu aktuellen datenschutzrechtlichen Themen interessiert sind. Ergebnisse und Vorträge der Konferenzen sind unter https://www.privacyos.eu abrufbar.

\section{Dr. Katalin Storf}

Projektleiterin PrivacyOS

Unabhängiges Landeszentrum für Datenschutz Schleswig-Holstein privacyos@datenschutzzentrum.de https://www.privacyos.eu 